\title{
Analysis on the genotoxicity of glyphosate using the theory of the electron transfer coefficient of quantum chemistry
}

\section{Análisis sobre la genotoxicidad del glifosato empleando la teoría del coeficiente de transferencia de electrones de química cuántica}

\author{
Manuel González Pérez ${ }^{1 *}$ \& Carmen Sánchez ${ }^{2}$
}

${ }^{1}$ Decanato de Biología y Posgrado en Ciencias de la Ingeniería Biomédica. Universidad Popular Autónoma del Estado de Puebla, Puebla, Mexico.

¿Laboratorio de Biotecnología, Centro de Investigación en Ciencias Biológicas, Universidad Autónoma de Tlaxcala. Tlaxcala, Mexico.

${ }^{*}$ Corresponding author

E-mail address: manuel.gonzalez@upaep.mx (M. González-Pérez)

Article history:

Received: 14 January 2020 / Received in revised form: 13 March 2019 / Accepted: 20 March 2020 / Published online: 26 March 2020.

https://doi.org/10.29267/mxjb.2020.5.1.43

\begin{abstract}
Glyphosate is the active substance in the herbicide, whose trademark is Roundup. This herbicide is used worldwide in weed control. There are a number of studies showing that glyphosate can cause genotoxicity/carcinogenicity. In this research, the genotoxicity of glyphosate was studied by making use of the theory of electron transfer coefficient (ETC) employing quantum chemical modeling. Quantum chemical molecular parameters were determined; the highest occupied molecular orbital (HOMO), lowest unoccupied molecular orbital (LUMO), the electrostatic potential $(E \delta)$, the positive partial molecular charge $(\delta+)$, the negative partial molecular charge $\left(\delta^{-}\right)$, the energy gap $(\mathrm{EBg})$, which were used to evaluate the ETC. The modeling was performed for the pure substance and for cross bands. Hyperchem software was used to calculate the ETC with the semi-empirical parametric method (SE-PM3). The software was calibrated with the algorithms of the quantum methods and their parameterization. The results showed that as a pure substance, glyphosate has the highest stability of all substances, so it can accumulate in biological systems. As cross bands, glyphosate initially oxidizes the nitrogen bases of DNA, followed by RNA. Quantum chemical modeling suggests that glyphosate is a carcinogen to biological systems, since it interacts with the nitrogenous bases of DNA, forming very stable molecules.
\end{abstract}


Keywords: Electron transfer coefficient, genotoxicity, glyphosate, nitrogen bases, quantum chemical modeling.

\section{RESUMEN}

Glifosato es el principio activo en el herbicida, cuya marca comercial es Roundup. Este herbicida es usado a nivel mundial en el control de maleza. Existen diversos estudios que muestran que el glifosato puede causar genotoxicidad/carcinogenicidad. En ésta investigación, se estudió la genotoxicidad del glifosato a través de la teoría del coeficiente de transferencia de electrones (ETC) usando modelamiento químico cuántico. Se determinaron los parámetros moleculares químicos cuánticos; el orbital molecular ocupado de más energía (HOMO), el orbital molecular no ocupado de más baja energía (LUMO), el potencial electrostático $(\mathrm{E} \delta)$, la carga molecular parcial positiva $(\delta+)$, la carga molecular parcial negativa $(\delta-)$, la energía de la banda prohibida $(\mathrm{EBg})$, mismos que fueron usados para evaluar el ETC. El modelamiento fue realizado para la sustancia pura y para bandas cruzadas. Se usó el software Hyperchem para el cálculo del ETC con el método paramétrico semi-empírico (SE-PM3). Se calibró el software con los algoritmos de los métodos cuánticos y su parametrización. Los resultados mostraron que como sustancia pura, el glifosato presenta la mayor estabilidad de todas las sustancias, por lo que puede acumularse en los sistemas biológicos. Como bandas cruzadas, el glifosato oxida inicialmente a las bases nitrogenadas del ADN, seguidas del ARN. El modelamiento químico cuántico sugiere que el glifosato es un agente cancerígeno para los sistemas biológicos, ya que interacciona con las bases nitrogenadas del ADN formando moléculas muy estables.

Palabras clave: Bases nitrogenadas, coeficiente de transferencia de electrones, genotoxicidad, glifosato, modelamiento químico cuántico.

\section{INTRODUCCIÓN}

El herbicida glifosato ( $\mathrm{N}$-(fosfonometil) glicina) es el principio más activo utilizado en todo el mundo para matar maleza de cultivos agrícolas (Aspelin, 1994). Este herbicida pertenece al grupo químico de los aminoácidos fosfonatos, cuya acción es inhibir la actividad de la enzima 5-enolpiruvil-shiquimato-3-fosfato sintetasa, que es un enzima importante en la síntesis de aminoácidos en algas, plantas y hongos (Schönbrunn et al., 2001). Existen diversos estudios que muestran que el glifosato y su principal metabolito ácido aminometilfosfónico, aparte de otros efectos tóxicos, puede causar genotoxicidad/carcinogenicidad (Paganelli, et al. 2010; Elie-Caille et al., 2010; George et al., 2010; Mañas et al., 2009a; Mañas et al., 2009b; Hultberg, 2007; Anadon et al., 2009; Peluso et al., 1988; Poletta et al., 2009; Benachour \& Seralini, 2009; Stur et al., 2019). La toxicidad de las formulaciones de la marca comercial de este herbicida (Roundup) ha sido evaluada en tres diferentes tipos de células humanas, en las cuales se mostró necrosis y apoptosis celular (Benachour \& Seralini, 2009). Samsel \& Seneff (2015) reportaron que el glifosato probablemente contribuye al aumento de múltiples tipos de cáncer en humanos. Por su parte, la Organización Mundial de la Salud ha clasificado al glifosato como "probablemente 
cancerígeno"

(Guyton

et

al.,

2015;

http://www.who.int/foodsafety/areas_work/chemical-risks/jmpr/en/). Por otro lado, la química cuántica tiene aplicaciones importantes en el estudio de sistemas biológicos (Ahuactzin-Peréz et al., 2016; González-Pérez, 2017a; 2017b; 2017c). La teoría orbital molecular extiende la teoría cuántica aplicada al modelo de estructuras químicas y afirma que los electrones están deslocalizados porque no están asignados a un átomo o enlace particular. En cambio, los electrones se mueven a través de la molécula en sus orbitales. Los orbitales moleculares son funciones de onda y contienen electrones de valencia entre sus átomos, que determinan la mayoría de las propiedades químicas de las moléculas. Esta teoría predice las propiedades espaciales y energéticas de los electrones para determinar las propiedades moleculares, y es la base para la mayoría de los métodos semiempíricos empleados en química cuántica (Živković, 1983). El método conocido como modelo semi-empírico parametrizado número 3 (SE-PM3) se ha utilizado para correlacionar parámetros químicos cuánticos moleculares y datos experimentales (Odunola \& Semire, 2007; González-Pérez et al., 2014). La visualización de las transformaciones químicas ayuda a obtener una comprensión fundamental más profunda sobre cómo se llevan a cabo las reacciones químicas. Esto es de utilidad para calcular los parámetros químicos cuánticos, así como el potencial electrostático de las moléculas, para entender el alineamiento molecular y para calcular el coeficiente de transferencia de electrones (ETC), mismo que ha sido usado en diversas investigaciones (González-Pérez et al., 2014; González-Pérez, 2015; González-Pérez et al., 2016a; .16b; Ahuatzin-Pérez et al., 2016; Balan-Rodríguez et al., 2016; Tejero-Jimenez et al., 2016; González-Pérez, 2017a; 2017b; 2017c; Vázquez-López et al., 2018; Flores-González et al., 2017; Pérez et al., 2019). El ETC indica el número de veces que el electrón necesita de su fuerza electromotriz natural para pasar del orbital molecular ocupado de más energía (HOMO) de una molécula al orbital molecular no ocupado de más baja energía (LUMO) de otra molécula, en una misma sustancia o entre sustancias diferentes (bandas cruzadas). En éste trabajo se determinaron los parámetros moleculares químicos cuánticos; el orbital molecular ocupado de más energía (HOMO), orbital molecular no ocupado de más baja energía (LUMO), el potencial electrostático (Eס), la carga molecular parcial positiva $\left(\delta^{+}\right)$, la carga molecular parcial negativa $\left(\delta^{-}\right)$, la energía de la banda prohibida $(\mathrm{EBg})$, mismos que fueron determinados para evaluar el ETC para el glifosato como sustancia pura y para las bandas cruzadas.

\section{MATERIALES Y MÉTODOS}

Los parámetros químicos cuánticos HOMO (electron Volts; eV), LUMO (eV), $\delta+$

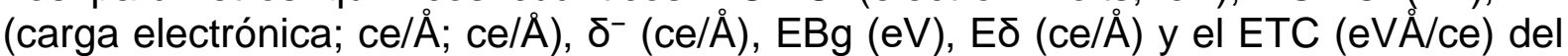
glifosafo fueron evaluados empleando el SE-PM3 (Odunola \& Semire, 2007; González-Pérez et al., 2014). El cálculo de los parámetros se realizó empleando las siguientes ecuaciones:

$\mathrm{EBg}=\mathrm{HOMO}-\mathrm{LUMO}$

$E \delta=\left|\left(\delta^{-}\right)-\left(\delta^{+}\right)\right|$

$\mathrm{ETC}=\mathrm{EBg} / \mathrm{E} \delta$ 
Los cálculos se realizaron usando un hardware Microsoft HP ATA (ST500DM002 IDB14SCSI.6.1.7600.16385). Se usó el software Hyperchem para el cálculo del ETC, empleando los parámetros específicos de las simulaciones previamente reportadas (González-Pérez et al., 2014).

\section{RESULTADOS}

En la Figura 1 se presentan las estructuras moleculares del glifosato, la molécula simple se muestra en la Figura 1a. En el primer momento de la reacción entre moléculas los polos negativos (maya roja) atraen a los polos positivos (maya azul). Los puntos neutros también se atraen (maya verde) (Fig. 1b). En el segundo momento (definitivo) el orbital HOMO de valencia (Fig. 1c) se acopla al orbital LUMO (Teoría Orbital Molecular) (Fig. 1d). Este acoplamiento permite que los electrones ubicados en HOMO salten al orbital LUMO formándose un enlace molecular. El glifosato es muy estable, debido a que el HOMO se sitúa en el átomo de nitrógeno; mientras que el LUMO se sitúa en el grupo fosfato y están muy cerca uno del otro. Si se juntan varias moléculas, se forma una esfera muy sólida, cuyo tamaño dependerá del número de coordinación molecular.
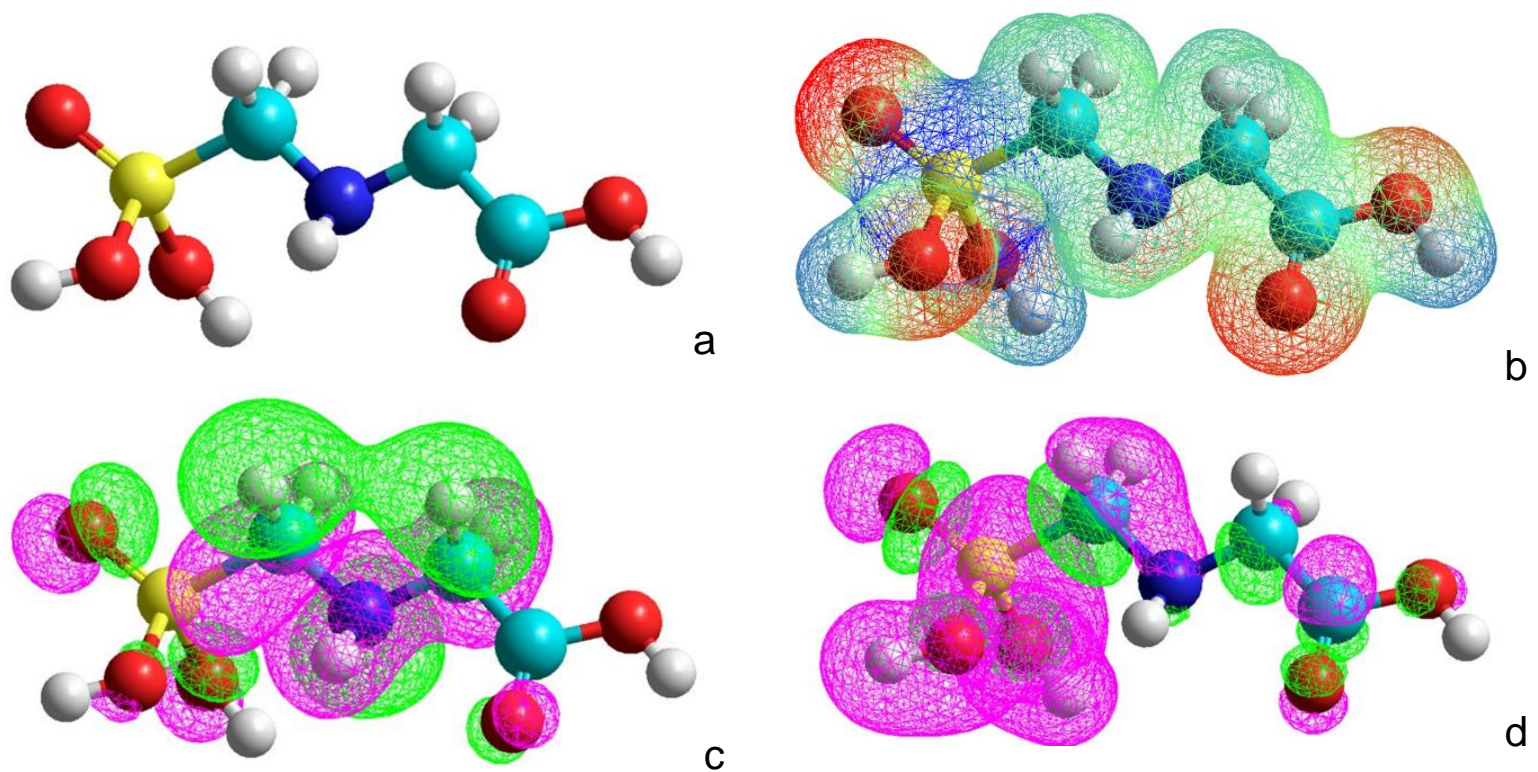

Fig. 1. Molécula de Glifosato en varias presentaciones; Molécula simple (a), Molécula con potencial electrostático (b), HOMO (c) y LUMO (d).

En la Figura 2 se muestra el pozo cuántico de las interacciones con glifosato vs base nitrogenada guanina. Se presentan cuatro interacciones de la siguiente manera; 1) Glifosato:Glifosato (línea azul), ésta es una interacción de la sustancia pura. 2) G:G (línea roja), ésta es la interacción de la sustancia pura de la base nitrogenada guanina. 3) Glifosato:G (punto verde), en ésta interacción el glifosato funciona como agente reductor (antioxidante) y se ubica en zona de baja probabilidad, y 4) G:Glisofato (punto rojo), en esta interacción el glifosato funciona como agente 
oxidante de la base nitrogenada guanina; éste punto se ubica en la zona de alta probabilidad y de mayor fuerza. El modelamiento químico cuántico mostró que en general, las tres bases nitrogenadas guanina, adenina y citosina presentaron (con pequeñas diferencias) el mismo patrón.

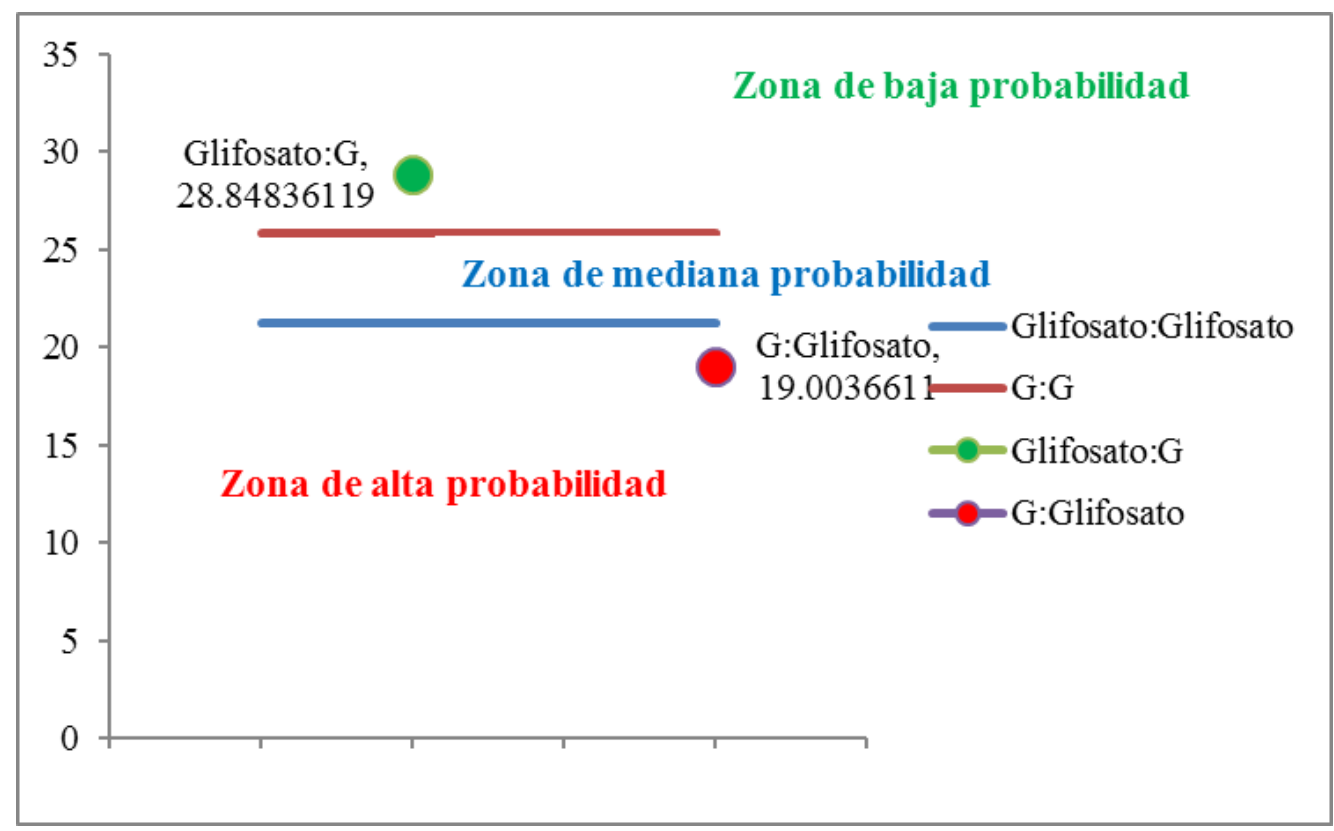

Fig. 2. Representación esquemática del pozo cuántico del glifosato con guanina (G). Se observa que la interacción G:Glifosato (punto rojo) se ubica en la zona de alta probabilidad.

En la Tabla 1 se muestran los cálculos del ETC de las bases nitrogenadas y del glifosato. Como se puede apreciar la última interacción corresponde a la interacción del glifosato, misma que muestra el menor valor de ETC, lo que indica que es la interacción más estable de todas ellas.

Tabla 1. ETC de las bases nitrogenadas.

\begin{tabular}{cccccccccc}
\hline $\mathbf{N}$ & Cede & Acepta & HOMO & LUMO & E $_{\text {BG }}$ & $\boldsymbol{\delta}^{-}$ & $\boldsymbol{\delta}^{+}$ & Eठ & ETC \\
\hline 1 & U1 & U1 & -9.71 & -0.511 & 9.2 & -0.126 & 0.171 & 0.297 & 30.975 \\
2 & T & T & -9.441 & -0.475 & 8.966 & -0.123 & 0.169 & 0.292 & 30.707 \\
3 & $\mathrm{~A}$ & $\mathrm{~A}$ & -8.654 & -0.213 & 8.441 & -0.14 & 0.156 & 0.296 & 28.518 \\
4 & $\mathrm{U} 2$ & $\mathrm{U} 2$ & -9.91 & -0.415 & 9.495 & -0.147 & 0.202 & 0.349 & 27.208 \\
5 & $\mathrm{C}$ & $\mathrm{C}$ & -9.142 & -0.344 & 8.799 & -0.174 & 0.161 & 0.335 & 26.265 \\
6 & $\mathrm{G}$ & $\mathrm{G}$ & -8.537 & -0.206 & 8.331 & -0.15 & 0.172 & 0.322 & 25.872 \\
7 & Glifosato & Glifosato & -9.870 & 0.452 & 10.322 & -0.163 & 0.323 & 0.486 & 21.239 \\
\hline
\end{tabular}

N: Numero de interacción. 
En la Tabla 2 se muestra el cruce de bandas entre las bases nitrogenadas y el glifosato, se puede observar los niveles de un solo pozo cuántico. En el fondo del pozo se encuentran las interacciones moleculares más estables (17-19). Conforme aumenta la profundidad del pozo, disminuye el valor de ETC (impedancia molecular). Las interacciones 17, 18 y 19 son las que muestran mayor afinidad química. Esto quiere decir que son las interacciones más probables y en las cuales la atracción molecular es mayor en comparación al resto de las interacciones. En éstas interacciones (17-19) se observa que el glifosato es un agente oxidante. Por otro lado, la interacción 16 también forma parte del fondo del pozo cuántico, lo que significa que el glifosato se queda como tal en el sistema biológico (es difícil de degradar).

Tabla 2. ETC de las bandas cruzadas del glifosato. En el fondo del pozo cuántico (17-19) se presentan las interacciones moleculares más estables.

\begin{tabular}{|c|c|c|c|c|c|c|c|c|c|}
\hline $\mathbf{N}$ & $\begin{array}{c}\text { Agente } \\
\text { Reductor }\end{array}$ & $\begin{array}{c}\text { Agente } \\
\text { Oxidante }\end{array}$ & & LUMO & $E_{B G}$ & $\delta^{-}$ & $\delta^{+}$ & Eס & ETC \\
\hline 1 & U1 & U1 & -9.71 & -0.511 & 9.2 & -0.126 & 0.171 & 0.297 & 30.975 \\
\hline 2 & $\mathrm{~T}$ & $\mathrm{~T}$ & -9.441 & -0.475 & 8.966 & -0.123 & 0.169 & 0.292 & 30.707 \\
\hline 3 & Glifosato & A & -9.870 & -0.213 & 9.657 & -0.163 & 0.156 & 0.319 & 30.273 \\
\hline 4 & Glifosato & C & -9.870 & -0.344 & 9.526 & -0.163 & 0.161 & 0.324 & 29.402 \\
\hline 5 & Glifosato & $\mathrm{G}$ & -9.870 & -0.206 & 9.664 & -0.163 & 0.172 & 0.335 & 28.848 \\
\hline 6 & A & A & -8.654 & -0.213 & 8.441 & -0.14 & 0.156 & 0.296 & 28.518 \\
\hline 7 & Glifosato & $\mathrm{T}$ & -9.870 & -0.475 & 9.395 & -0.163 & 0.169 & 0.332 & 28.299 \\
\hline 8 & Glifosato & U1 & -9.870 & -0.511 & 9.359 & -0.163 & 0.171 & 0.334 & 28.022 \\
\hline 9 & U2 & U2 & -9.91 & -0.415 & 9.495 & -0.147 & 0.202 & 0.349 & 27.208 \\
\hline 10 & C & C & -9.142 & -0.344 & 8.799 & -0.174 & 0.161 & 0.335 & 26.265 \\
\hline 11 & Glifosato & U2 & -9.870 & -0.415 & 9.455 & -0.163 & 0.202 & 0.365 & 25.905 \\
\hline 12 & G & $\mathrm{G}$ & -8.537 & -0.206 & 8.331 & -0.15 & 0.172 & 0.322 & 25.872 \\
\hline 13 & U1 & Glifosato & -9.71 & 0.452 & 10.162 & -0.126 & 0.323 & 0.449 & 22.632 \\
\hline 14 & $\mathrm{~T}$ & Glifosato & -9.441 & 0.452 & 9.893 & -0.123 & 0.323 & 0.446 & 22.181 \\
\hline 15 & U2 & Glifosato & -9.91 & 0.452 & 10.362 & -0.147 & 0.323 & 0.470 & 22.046 \\
\hline 16 & Glifosato & Glifosato & -9.870 & 0.452 & 10.322 & -0.163 & 0.323 & 0.486 & 21.239 \\
\hline 17 & A & Glifosato & -8.654 & 0.452 & 9.106 & -0.14 & 0.323 & 0.463 & 19.667 \\
\hline 18 & C & Glifosato & -9.142 & 0.452 & 9.594 & -0.174 & 0.323 & 0.497 & 19.303 \\
\hline 19 & $G$ & Glifosato & -8.537 & 0.452 & 8.989 & -0.15 & 0.323 & 0.473 & 19.004 \\
\hline
\end{tabular}

$\mathbf{N}$ : Numero de interacción. 
En la Tabla 3 se muestran las interacciones 1-4, mismas que son permitidas por la naturaleza y las interacciones anormales que se presentan entre el herbicida glifosato y las bases nitrogenadas (5-11). Como se puede apreciar las interacciones anormales son más afines que las interacciones naturales. La interacción 5, corresponde a un U1 (uracilo en su tautómero cetónico). Esta interacción es la más inestable de todas. La interacción 7 corresponde a U2 (uracilo en su tautómero hidroxilado), misma que tiene una estabilidad intermedia, siendo mejor que el tautómero U1. Por lo anterior, se puede concluir que el tautómero más abundante en el ácido desoxirribonucleico (ARN) es el U2 (Tabla 1).

Tabla 3. ETC de las interacciones entre bases nitrogenadas permitidas en la naturaleza (1-4) y de las interacciones entre las bases nitrogenadas y el glifosato (511, interacciones moleculares más estables, anormales y oxidativas del glifosato).

\begin{tabular}{|c|c|c|c|c|c|}
\hline $\mathbf{N}$ & Reductor & Oxidante & ETC & $\begin{array}{l}\text { Molécula } \\
\text { formada }\end{array}$ & Característica de la interacción \\
\hline 1 & A & $\mathrm{T}$ & 26.471 & ADN & $\begin{array}{llll}\text { Interacción } & \text { permitida } & \text { por } & \text { la } \\
\text { naturaleza. } & & & \end{array}$ \\
\hline 2 & A & U1 & 26.185 & ARN & $\begin{array}{l}\text { Interacción permitida por la } \\
\text { naturaleza. }\end{array}$ \\
\hline 3 & C & $G$ & 25.827 & ADN & $\begin{array}{l}\text { Interacción permitida por la } \\
\text { naturaleza. }\end{array}$ \\
\hline 4 & $A$ & U2 & 24.092 & ARN & $\begin{array}{l}\text { Interacción permitida por la } \\
\text { naturaleza. }\end{array}$ \\
\hline 5 & U1 & Glifosato & 22.632 & ARN & $\begin{array}{l}\text { ARN "contaminado" con el } \\
\text { tautómero cetónico del glifosato. }\end{array}$ \\
\hline 6 & $\mathrm{~T}$ & Glifosato & 22.181 & ADN, ARN & $\begin{array}{l}\text { ADN y ARN "contaminados" con } \\
\text { glifosato. }\end{array}$ \\
\hline 7 & U2 & Glifosato & 22.046 & ARN & $\begin{array}{l}\text { ARN "contaminado" con el } \\
\text { tautómero hidroxilado } \\
\text { glifosato. }\end{array}$ \\
\hline 8 & Glifosato & Glifosato & 21.239 & glifosato & $\begin{array}{l}\text { Acumulación del glifosato en el } \\
\text { sistema biológico. }\end{array}$ \\
\hline 9 & $A$ & Glifosato & 19.667 & ADN & $\begin{array}{l}\text { El glifosato "contamina" al } \\
\text { con mucha atracción y alta } \\
\text { probabilidad. }\end{array}$ \\
\hline 10 & C & Glifosato & 19.303 & ADN & $\begin{array}{l}\text { El glifosato "contamina" al } \text { ADN } \\
\text { con mucha atracción y alta } \\
\text { probabilidad. }\end{array}$ \\
\hline 11 & $G$ & Glifosato & 19.004 & ADN & $\begin{array}{l}\text { El glifosato "contamina" al ADN } \\
\text { con mucha atracción y alta } \\
\text { probabilidad. }\end{array}$ \\
\hline
\end{tabular}

$\mathbf{N}$ : Numero de interacción. 


\section{DISCUSIÓN}

Como se muestra previamente son siete interacciones de sustancias puras que se llevan a cabo (una interacción del glifosato y seis de las bases nitrogenadas), siendo la interacción del glifosato la más estable por ser la más pequeña de todas (Tabla 1). Lo anterior implica que si el humano está expuesto al glifosato y éste se introduce al cuerpo humano, el glifosato se mantendrá como sustancia pura. El hecho de que el glifosato se encuentre en el fondo del pozo cuántico muestra que es muy difícil que el cuerpo humano sea capaz de degradar este herbicida a través de la acción de alguna enzima u otro tipo de mecanismo, ya que la interacción molecular del herbicida es muy estable. Lo anterior, cumple el principio de la mínima acción de Feynmann (Gray, 2018), es decir, las reacciones en la naturaleza tienden a emplear mínima energía. Un ETC más bajo presenta un menor obstáculo al electrón en su trayectoria (valencia de enlace). Esto implica que el glifosato puede romper al ADN y al ARN como blanco principal y secundario, respectivamente, y eventualmente puede también dañar al ATP. Estos resultados confirman lo reportado previamente sobre los daños causados en la actividad de los espermatozoides humanos debido a la acumulación de glifosato en mamíferos (Cardona-Maya, 2019; Pham et al., 2019). Los resultados obtenidos en ésta investigación muestran que las moléculas del glifosato pueden reaccionar más fácilmente con las bases nitrogenadas (interacciones 17-19), que incluso con otras moléculas de glifosato (interacción 16), ya que el valor del ETC es mayor en la interacción entre moléculas de glifosato que entre moléculas del glifosato y bases nitrogenadas (Tabla 2). Stur et al. (2019) reportaron que el glifosato puede causar daño celular a dosis bajas en un período de tiempo relativamente corto, afectando principalmente el ciclo celular y la reparación del ADN. El modelamiento químico cuántico sugiere que el glifosato es un agente cancerígeno para los sistemas biológicos. Éste efecto cancerígeno se debe a que el glifosato interacciona con las bases nitrogenadas tanto del ADN como del ARN formando conglomerados moleculares más estables que las interacciones naturales permitidas, ya que el glifosato actúa como agente oxidante en todas las bases nitrogenadas.

\section{REFERENCIAS}

Ahuactzin-Pérez M., Tlecuitl-Beristain S., García-Dávila J., González-Pérez M., Gutiérrez-Ruíz, M. C. \& Sánchez C. 2016. Degradation of di (2-ethyl hexyl) phthalate by Fusarium culmorum: kinetics, enzymatic activities and biodegradation pathway based on quantum chemical modeling. Science of the Total Environment. 566-567: 1186-1193.

Anadon A., Martinez-Larranaga M. R., Martinez M. A., Castellano V. J., Martinez M., Martin M.T., Nozal M. J., Bernal J. L. 2009. Toxicokinetics of glyphosate and its metabolite aminomethyl phosphonic acid in rats, Toxicology Letters. 190: 91-95.

Aspelin A. L. 1994. Pesticide industry sales and usage: 1992 and 1993 market estimates. Washington (DC): U.S. EPA. Office of Prevention, Pesticides and Toxic Substances. Office of Pesticide Programs. Biological and Economic Analysis Division. 
Balan-Rodriguez L., Tejero-Jimenez L., Loya-Vargas M. D. \& González-Pérez M. 2016. Research carcinogenic effect of tartrazine vs nitrogenous bases by the quantum method (zindo/1). World Journal of Pharmaceutical Research. 5(8): 241248.

Benachour N. \& Seralini G. E. 2009. Glyphosate formulations induce apoptosis and necrosis in human umbilical, embryonic, and placental cells. Chemical Research in Toxicology. 22: 97-105.

Cardona-Maya W. D. 2019. El glifosato afecta negativamente a los espermatozoides humanos: evidencia in vitro. Revista Urología Colombiana. doi:10.1055/s-00391696699.

Elie-Caille C., Heu C., Guyon C. \& Nicod L. 2010. Morphological damages of a glyphosate-treated human keratinocyte cell line revealed by a micro- to nanoscale microscopic investigation. Cell Biology and Toxicology. 26(4): 331-339.

Flores-González A. L. Miguel Gutiérrez-Ramírez J., Sánchez-González L. C., ReyesSolis H., Toledo-Vázquez A., González-Pérez M. 2017. Quantic analysis of the effect of nicotine on neurotransmitters. World Journal of Pharmaceutical Research 6(4): 317-326.

George J., Prasad S., Mahmood Z. \& Shukla Y. 2010. Studies on glyphosate-induced carcinogenicity in mouse skin: a proteomic approach. Journal of Proteomics. 73: 951-964.

González-Pérez M. 2015. Applied quantum chemistry: Analysis of the rules of Markovnikov and anti-Markovnikov. International Journal of Science and Advanced Technology. 5: 5.

González-Pérez M. 2016a. Interactions analysis of four chemotherapeutic drugs vs. nitrogenous bases of DNA and RNA, using quantum methods. World Journal of Pharmaceutical Research. 5(6): 309-320.

González-Pérez M. 2017a. Quantum theory of the electron transfer coefficient. International Journal of Advanced Engineering, Management and Science. 3: 10.

González-Pérez M. 2017b. Quantum modeling to determine the carcinogenic potential of aflatoxin B1 produced by Aspegillus $\mathrm{sp}$ and its metabolic derivate aflatoxin M1. Mexican Journal of Biotechnology. 2(2): 255-270.

González-Pérez M. 2017c. Chemical-quantum analysis of the aggressiveness of glucose and its appeasement with ATP inside the cell, and water as an excellent antioxidant. World Journal of Pharmacy and Pharmaceutical Sciences. 6(4): 95-99.

González-Pérez M., Briteño-Vázquez M., García-Barrera F. A., Ham-Tirado A. K., López-Oglesby J. M., Salazar-Amador M. R., Pacheco-García P.F. 2016b. Molecular interactions of nicotine and the nitrogenous bases of DNA and RNA calculated by 
improved quantum methods. World Journal of Pharmaceutical Research. 5(3): 17781792.

González-Pérez, M., García-Barrera A., Antonio F., Méndez-Díaz J. F., GonzálezTorres M. \& López-Oglesby J. M. 2014. Theoretical calculation of electron transfer coefficient for predicting the flow of electrons by PM3, using 20 amino acids and nicotine. European Scientific Journal 10(27): 42-49.

Gray C. G. 2018. The lazy universe: An introduction to the principle of least action. Coopersmith Jennifer. 279 pp. Oxford U.P., New York.

Guyton K. Z., Loomis D., Grosse Y., El Ghissassi F., Benbrahim-Tallaa L., Guha N., Scoccianti C., Mattock H. \& Straif K.. 2015. On behalf of the International Agency for Research on Cancer Monograph Working Group, IARC, Lyon, France. Carcinogenicity of tetrachlorvinphos, parathion, malathion, diazinon, and glyphosate. The Lancet. 16: 490-491.

Hultberg M. 2007. Cysteine turnover in human cell lines is influenced by glyphosate, Environmental Toxicology and Pharmacology. 24: 19-22.

Mañas F., Peralta L., Raviolo J., García Ovando H., Weyers A., Ugnia L., GonzalezCid M., Larripa I. \& Gorla N. 2009a. Genotoxicity of AMPA, the environmental metabolite of glyphosate, assessed by the Comet assay and cytogenetic tests. Ecotoxicology and Environmental Safety. 72. 834-837.

Mañas F., Peralta L., Raviolo J., Ovando H. G., Weyers A., Ugnia L., Cid M. G., Larripa I., Gorla N. 2009b. Genotoxicity of glyphosate assessed by the comet assay and cytogenetic tests. Environmental Toxicology and Pharmacology. 28(1): 37-41.

Odunola O. A. \& Semire B. 2007. Conformational analysis (Semi Empirical PM3) and electronic properties of functionalized oligo (hexylpyrroles). Electronic Journal of Chemistry. 4(3): 363-371.

Paganelli A., Gnazzo V., Acosta H., López S. L. \& Carrasco A. E. 2010. Glyphosatebased herbicides produce teratogenic effects on vertebrates by impairing retinoic acid signaling. Chemical Research in Toxicology. 23(10): 1586-1595.

Peluso M., Munnia A., Bolognesi C. \& Parodi S. 1998. ${ }^{32}$ P-postlabeling detection of DNA adducts in mice treated with the herbicide roundup. Environmental and Molecular Mutagenesis. 31(1): 55-59.

Pérez M. G., Soria V. R. \& Mioni L. C. 2019. Demonstration of the formation of the caffeine-dichloromethane-water emulsion using quantum chemistry. International Journal of Advanced Engineering, Management and Science. 4(11): 782-785.

Pham T. H., Derian L., Kervarrec C., Kernanec P. Y., Jégou B., Smagulova F. \& Gely-Pernot A. 2019. Perinatal exposure to glyphosate and a glyphosate-based herbicide affect spermatogenesis in mice. Toxicological Sciences. 169(1): 260-271. 
Poletta G. L., Larriera A., Kleinsorge E. \& Mudry M. D. 2009. Genotoxicity of the herbicide formulation Roundup ${ }^{\circledR}$ (glyphosate) in broad-snouted caiman (Caiman latirostris) evidenced by the Comet assay and the Micronucleus test. Mutation Research/Genetic Toxicology and Environmental Mutagenesis. 672(2): 95-102.

Samsel A. \& Seneff S. 2015. Glyphosate, pathways to modern diseases IV: cancer and related pathologies. Journal of Biological Physics and Chemistry. 15: 121-159.

Schönbrunn, E., Eschenburg S., Shuttleworth W. A., Schloss J. V., Amrhein N., Evans J. N. S. \& Kabsch W. 2001. Interaction of the herbicide glyphosate with its target enzyme 5-enolpyruvylshikimate 3-phosphate synthase in atomic detail. Proceedings of the National Academy of Sciences. 98(4): 1376-1380.

Stur E., Aristizabal-Pachon A. F., Peronni K. C., Agostini L. P., Waigel S., Chariker J., Miller D. M., Thomas S. D., Rezzoug F., Detogni R. S., Reis R. S. D., Silva Junior W. A. \& Louro I. D. 2019. Glyphosate-based herbicides at low doses affect canonical pathways in estrogen positive and negative breast cancer cell lines. PLoS One. 14(7): e0219610.

Tejero-Jimenez L., Balan-Rodriguez L, Loya-Vargas M. D., González-Pérez M. 2016. Analysis of interactions of neotame and nitrogenous bases of DNA and RNA using quantum methods. World Journal of Pharmaceutical Research 5(8): 241-248.

Vázquez-López E., Pedraza-Gress E., García-Aguilar K., Herrera-Cantú I., GarcíaMar J. J., Flores-González L. A., Aparicio-Razo M., Sánchez-Parada O. \& GonzálezPérez, M. (2018). Quantum modeling to determine the carcinogenic potential of aflatoxin b2 produced by Aspergillus flavus and Aspergillus parasiticus. 7:4 World Journal of Pharmacy and Pharmaceutical Sciences.7:4 243-251.

Živković, T. P. 1983. Molecular orbital resonance theory: A new approach to the treatment of quantum chemical problems. Theoretica Chimica Acta. 62(4): 335-350. 